\title{
Neutrino mass hierarchy and three-flavor spectral splits of supernova neutrinos
}

\author{
Basudeb Dasgupta, ${ }^{1}$ Alessandro Mirizzi, ${ }^{2}$ Irene Tamborra,,${ }^{1,3,4}$ and Ricard Tomàs ${ }^{2}$ \\ ${ }^{1}$ Max-Planck-Institut für Physik (Werner-Heisenberg-Institut), Föhringer Ring 6, 80805 München, Germany \\ ${ }^{2}$ II Institut für Theoretische Physik, Universität Hamburg, \\ Luruper Chaussee 149, 22761 Hamburg, Germany \\ ${ }^{3}$ Dipartimento Interateneo di Fisica "Michelangelo Merlin", Via Amendola 173, 70126 Bari, Italy \\ ${ }^{4}$ INFN, Sezione di Bari, Via Orabona 4, 70126 Bari, Italy
}

(Dated: October 29, 2018)

\begin{abstract}
It was recently realized that three-flavor effects could peculiarly modify the development of spectral splits induced by collective oscillations, for supernova neutrinos emitted during the cooling phase of a protoneutron star. We systematically explore this case, explaining how the impact of these three-flavor effects depends on the ordering of the neutrino masses. In inverted mass hierarchy, the solar mass splitting gives rise to instabilities in regions of the (anti)neutrino energy spectra that were otherwise stable under the leading two-flavor evolution governed by the atmospheric mass splitting and by the 1-3 mixing angle. As a consequence, the high-energy spectral splits found in the electron (anti)neutrino spectra disappear, and are transferred to other flavors. Imperfect adiabaticity leads to smearing of spectral swap features. In normal mass hierarchy, the three-flavor and the two-flavor instabilities act in the same region of the neutrino energy spectrum, leading to only minor departures from the two-flavor treatment.
\end{abstract}

PACS numbers: 14.60.Pq, 97.60.Bw

\section{INTRODUCTION}

The neutrino flux from a core-collapse supernova $(\mathrm{SN})$ is a powerful tool to probe fundamental neutrino properties as well as the dynamics of the explosion [1, 2]. The diagnostic role played by neutrinos during a stellar collapse is largely associated with the signatures imprinted on the observable SN neutrino burst by flavor conversions occurring deep inside the star.

It has been understood that the paradigm of neutrino flavor transformation in supernovae [3], based primarily on the Mikheyev-Smirnov-Wolfenstein (MSW) effect with the ordinary matter [4], was incomplete. New surprising and unexpected effects have been found to be important in the region close to the neutrinosphere (see [5, 6] for recent reviews). Here the neutrino density is so high that the neutrino-neutrino interactions dominate the flavor evolution, producing collective oscillations. The most important observational consequence of $\nu-\nu$ interactions is a swap of the $\nu_{e}$ and $\bar{\nu}_{e}$ spectra with the non-electron $\nu_{x}$ and $\bar{\nu}_{x}$ spectra in certain energy ranges $[7,[8]$.

The development of the spectral swaps is strongly dependent on the original SN neutrino fluxes. In the accretion phase, i.e. $t \lesssim 0.5 \mathrm{~ms}$ after the core-bounce, neutrino number fluxes are expected to be ordered as $\left.\Phi_{\nu_{e}}^{0}>\Phi_{\bar{\nu}_{e}}^{0} \gg \Phi_{\bar{\nu}_{x}}^{0}[9] 11\right]$. In such a scenario, one finds that for normal neutrino mass hierarchy (NH, $\left.\Delta m_{\mathrm{atm}}^{2}=m_{3}^{2}-m_{1,2}^{2}>0\right)$ collective oscillations do not play a significant role. For inverted hierarchy (IH, $\left.\Delta m_{\mathrm{atm}}^{2}<0\right)$, the end of collective oscillations is marked by a complete exchange of the $e$ and $x$ flavors for almost all antineutrinos. For neutrinos, the exchange happens only above a characteristic energy fixed by the lepton number conservation, giving rise to a spectral split in their energy distributions 12 15].

The neutrino number fluxes may be significantly different at later times, i.e. during the cooling phase. In Garching simulations [1], one finds a cross-over among the different $\nu$ spectra. As a consequence, the original fluxes exhibit a different ordering $\Phi_{\nu_{x}}^{0} \gtrsim \Phi_{\nu_{e}}^{0} \gtrsim \Phi_{\bar{\nu}_{e}}^{0}$. A study of this latter case was performed [16], finding the occurrence of unexpected multiple spectral splits for both neutrinos and antineutrinos, in normal and inverted mass hierarchies. The rich phenomenology of the spectral splits, and its dependence on the original neutrino energy spectra was further explored in the extensive study performed in [17].

Most of the collective flavor dynamics of SN neutrinos can be explained in an effective two-flavor $(2 \nu)$ framework [18]. Collective oscillations are triggered by an instability in the two-flavor " $H$-sector" associated with the atmospheric mass-squared difference $\Delta m_{\text {atm }}^{2}$ and the mixing angle $\theta_{13}$. Three-flavor $(3 \nu)$ effects are due to the " $L$-sector," governed by the smaller solar mass splitting $\Delta m_{\mathrm{sol}}^{2}=m_{2}^{2}-m_{1}^{2}>0$ and the mixing angle $\theta_{12}$. These effects have been studied for neutrino fluxes typical of the accretion phase [18 21]. It was recently shown 22] that they are able to trigger collective flavor conversions, even if the mixing angle $\theta_{13}$ is exactly zero. However, apart from this initial kick, no new sizeable effect was found in the subsequent neutrino flavor evolution.

Recently, the $3 \nu$ case was studied for a scenario relevant to the cooling phase 23]. It was found that in the inverted mass hierarchy, the presence of the solar sector can "erase" the high-energy spectral splits that would have occurred in the $\nu_{e}$ and $\bar{\nu}_{e}$ spectra for a $2 \nu$ flavor evolution governed by the $\Delta m_{\text {atm }}^{2}$ and $\theta_{13}$. Moreover, the final electron antineutrino energy spectrum exhibits a "mixed" nature, i.e. the spectral 
swap is not complete. The phenomenological importance of this result is underlined by the fact that the highenergy spectral features are expected to be significantly easier to observe at neutrino detectors. In [23] these three-flavor effects are associated with an instability in the $L$ sector and to the subsequent non-adiabatic flavor evolution driven by $\Delta m_{\mathrm{sol}}^{2}$. Building on this insight, we find it worthwhile to take a closer look at the three-flavor effects during the cooling phase, and to understand the origin and nature of the $3 \nu$ instability.

We explain how the $3 \nu$ effects are crucially dependent on the neutrino mass hierarchy. For normal mass hierarchy, there is no fundamental difference between $H$ and $L$ sectors, since in this case both mass splittings are positive and the effective in-medium mixing angles are both small. Thus, even in a $2 \nu$ set-up one would expect spectral splits driven by the $L$-sector parameters, and expect them to be exactly where the splits appear in the case of $H$-sector in normal hierarchy. However we find that, it is not the case. In fact with typical SN parameters, collective oscillations fail to produce any significant conversions for the $L$-sector. This is so, because the $L$-sector has a lower natural frequency ( $\omega_{L}=\Delta m_{\mathrm{sol}}^{2} / 2 E$, where $E$ is a typical SN $\nu$ energy) than the $H$-sector and the collective interaction strength drops at a rate much faster than it. This does not leave enough time for the instability to grow and makes the collective evolution non-adiabatic. Therefore, spectral splits fail to develop. Nevertheless, the system is in an unstable situation. Indeed, as we will show, a small perturbation in the initial conditions is enough to develop the collective flavor conversions, producing spectral swaps also in this two-flavor case.

In a realistic situation, this initial perturbation for the $L$-sector is provided by $3 \nu$ effects that couple this sector to the $H$-sector. Oscillations in the $H$-sector are communicated to the $L$-sector, allowing the instability to grow much faster. The spectral swapping still remains less adiabatic than in the $H$-sector. As a result, for normal mass hierarchy - where both $H$ and $L$-sectors trigger the same instability and "compete" to convert the high-energy $\nu$ and $\bar{\nu}$ spectra - the $H$-sector wins. Threeflavor effects do not cause a significant change. The interesting bit happens for inverted mass hierarchy. In this case, the $H$-sector and the $L$-sector have instabilities in different parts of the spectrum and therefore do not compete with each other. Instead, they "cooperate" and act on complementary parts of the energy spectra. The $L$-sector instability catalyzed by the $H$-sector, operates in the high-energy region without hindrance, and causes an additional swap, that erases the spectral split found in the $2 \nu$ flavor evolution. The low adiabaticity in the $L$-sector is responsible for somewhat smeared splits, and the effect is particularly important for splits at higher energies, especially for the antineutrinos. In the remainder of this paper, we illustrate these aspects using simple examples and provide a semi-analytical treatment.

The plan of our work is as follows. In Section 2, we present our formalism for the SN neutrino flavor evolution and set up our numerical calculations, i.e. state our inputs for neutrino masses, mixing parameters, original supernova neutrino energy spectra and luminosities at late times. In Section 3, we present the $2 \nu$ results in the $H$ and $L$ sectors, in particular showing the lack of adiabaticity in the $L$-sector and the role of the small perturbations in the initial conditions to circumvent that. In Section 4, we present a complete $3 \nu$ calculation, showing that the $H$-sector catalyzes the $L$-sector and then instabilities in both sectors develop - in cooperation for inverted mass hierarchy, and in competition for normal mass hierarchy. We provide an estimates of the relative adiabaticity of the low energy and high energy splits - explaining the mixed spectra observed in the antineutrino sector. Finally in Section 5, we comment on our results and conclude.

\section{EQUATIONS OF MOTION AND NUMERICAL FRAMEWORK}

Mixed neutrinos are described by matrices of density $\rho_{\mathbf{p}}$ and $\bar{\rho}_{\mathbf{p}}$ for each (anti)neutrino mode. The diagonal entries are the usual occupation numbers whereas the offdiagonal terms encode phase information. The equations of motion (EoMs) are [8, 24]

$$
\mathrm{i} \partial_{t} \rho_{\mathbf{p}}=\left[\mathbf{H}_{\mathbf{p}}, \rho_{\mathbf{p}}\right],
$$

where the Hamiltonian is

$$
\mathrm{H}_{\mathbf{p}}=\Omega_{\mathbf{p}}+V+\sqrt{2} G_{F} \int \frac{\mathrm{d}^{3} \mathbf{q}}{(2 \pi)^{3}}\left(\rho_{\mathbf{q}}-\bar{\rho}_{\mathbf{q}}\right)\left(1-\mathbf{v}_{\mathbf{q}} \cdot \mathbf{v}_{\mathbf{q}}\right),
$$

$\mathbf{v}_{\mathbf{p}}$ being the velocity. The matrix of the vacuum oscillation frequencies for neutrinos is $\Omega_{\mathbf{p}}=\left(m_{1}^{2}, m_{2}^{2}, m_{3}^{2}\right) / 2|\mathbf{p}|$ in the mass basis. For antineutrinos $\Omega_{\mathbf{p}} \rightarrow-\Omega_{\mathbf{p}}$. The matter effect due to the background electron density $n_{e}$ is represented, in the weak-interaction basis, by $V=$ $\sqrt{2} G_{F} n_{e} \operatorname{diag}(1,0,0)$.

In spherical symmetry the EoMs can be expressed as a closed set of differential equations along the radial direction [25, 26] . The factor $\left(1-\mathbf{v}_{\mathbf{q}} \cdot \mathbf{v}_{\mathbf{q}}\right)$ in the Hamiltonian, implies "multi-angle" effects for neutrinos moving on different trajectories [7]. However, for realistic supernova conditions the modifications are small, allowing for a single-angle approximation [20, 25]. We implement this approximation by launching all neutrinos with $45^{\circ}$ relative to the radial directions [19, 25].

For the numerical illustrations, we take the neutrino mass-squared differences in vacuum to be $\Delta m_{\mathrm{atm}}^{2}=$ $2 \times 10^{-3} \mathrm{eV}^{2}$ and $\Delta m_{\mathrm{sol}}^{2}=8 \times 10^{-5} \mathrm{eV}^{2}$, close to their current best-fit values [27]. The values of the mixing parameters relevant for SN neutrino flavor conversions are $\sin ^{2} \theta_{12} \simeq 0.31$ and $\sin ^{2} \theta_{13} \lesssim 0.04$ [27]. The matter effect in the region of collective oscillations (up to a few $100 \mathrm{~km}$ ) can be accounted for by choosing small (matter suppressed) mixing angles [28], which we take to be $\tilde{\theta}_{13}=$ 
$\tilde{\theta}_{12}=10^{-3}$, and considering as effective mass-square differences in matter $\Delta \tilde{m}_{\mathrm{atm}}^{2}=\Delta m_{\mathrm{atm}}^{2} \cos \theta_{13} \simeq \Delta m_{\mathrm{atm}}^{2}$ and $\Delta \tilde{m}_{\mathrm{sol}}^{2}=\Delta m_{\mathrm{sol}}^{2} \cos 2 \theta_{12} \simeq 0.4 \Delta m_{\mathrm{sol}}^{2}[28,29]$. We ignore possible subleading $\mathrm{CP}$ violating effects [21] by setting $\delta_{\mathrm{CP}}=0$. MSW conversions typically occur after collective effects have ceased [20, 30]. Their effects then factorize and can be included separately. Therefore, we neglect them in the following.

The content of a given neutrino species $\nu_{\alpha}$ with energy $E$ at a position $r$ is given by

$$
\rho_{\alpha \alpha}(E, r)=\frac{F_{\nu_{\alpha}}(E, r)}{F(E, r)}
$$

where $F_{\nu_{\alpha}}$ is the energy distribution of $\nu_{\alpha}$ and $F$ is the sum of the energy distributions of all flavors, for neutrinos and antineutrinos respectively. For the three relevant SN $\nu$ energy distributions at the neutrinosphere $F_{\nu_{\alpha}}^{0}$, we take

$$
F_{\nu_{\alpha}}^{0}(E)=\Phi_{\nu_{\alpha}}^{0} \varphi_{\nu_{\alpha}}(E)
$$

where $\Phi_{\nu_{\alpha}}^{0}=L_{\nu_{\alpha}} /\left\langle E_{\nu_{\alpha}}\right\rangle$ is the number flux, defined in terms of the neutrino luminosity $L_{\nu_{\alpha}}$ and the neutrino average energy $\left\langle E_{\nu_{\alpha}}\right\rangle \cdot \varphi_{\nu_{\alpha}}(E)$ is the normalized neutrino spectrum $\left(\int d E \varphi_{\nu_{\alpha}}=1\right)$, parametrized as [11]:

$$
\varphi_{\nu_{\alpha}}(E)=\frac{\beta^{\beta}}{\Gamma(\beta)} \frac{E^{\beta-1}}{\left\langle E_{\nu_{\alpha}}\right\rangle^{\beta}} e^{-\beta E /\left\langle E_{\nu_{\alpha}}\right\rangle},
$$

where $\beta$ is a spectral parameter, and $\Gamma(\beta)$ is the Euler gamma function. The values of the parameters are model dependent [9, 10]. For our numerical illustrations, we choose

$$
\left(\left\langle E_{\nu_{e}}\right\rangle,\left\langle E_{\bar{\nu}_{e}}\right\rangle,\left\langle E_{\bar{\nu}_{x}}\right\rangle\right)=(12,15,18) \mathrm{MeV},
$$

and $\beta=4$, from the admissible parameter ranges [1]. We take ratios of the fluxes at late-times to be [10]

$$
\Phi_{\nu_{e}}^{0}: \Phi_{\bar{\nu}_{e}}^{0}: \Phi_{\nu_{x}}^{0}=0.85: 0.75: 1.0
$$

where we have assumed equal $\mu$ and $\tau$ neutrino and antineutrino initial fluxes.

The strength of the neutrino-neutrino interactions can be parametrized as 25]

$$
\mu_{0}=\sqrt{2} G_{F}\left|F_{\bar{\nu}_{e}}^{0}-F_{\bar{\nu}_{x}}^{0}\right|,
$$

where the fluxes are taken at the neutrinosphere radius $R=10 \mathrm{~km}$. Numerically, we assume $\mu_{0}=7 \times 10^{5} \mathrm{~km}^{-1}$. When formally deriving the single-angle approximation, one explicitly obtains that the radial dependence of the neutrino-neutrino interaction strength can be written as 26.

$$
\mu(r)=\mu_{0} \frac{R^{2}}{r^{2}} C_{r},
$$

where the $r^{-2}$ scaling comes from the geometrical flux dilution, and the collinearity factor

$$
\begin{aligned}
C_{r} & =4\left[\frac{1-\sqrt{1-(R / r)^{2}}}{(R / r)^{2}}\right]^{2}-1 \\
& =\frac{1}{2}\left(\frac{R}{r}\right)^{2} \quad \text { for } r \rightarrow \infty,
\end{aligned}
$$

arises from the $(1-\cos \theta)$ structure of the neutrinoneutrino interaction. The asymptotic behavior for large $r$ agrees with what one obtains by considering that all neutrinos are launched at $45^{\circ}$ to the radial-direction [19]. The known decline of the neutrino-neutrino interaction strength, $\mu(r) \sim r^{-4}$ for $r \gg R$, is evident.

\section{MULTIPLE SPECTRAL SPLITS IN A TWO-FLAVOR SCENARIO}

\section{A. $2 \nu H$-system}

We start our investigation with the flavor evolution in the $H$-sector characterized by $\left(\Delta m_{\mathrm{atm}}^{2}, \theta_{13}\right)$. As explained in [16], spectral swaps can develop around energies $E=E_{c}$ of the original neutrino spectra (except at $E=0$ ), where spectra of different flavors cross each other, i.e. at the crossing points

$$
\begin{aligned}
& F_{\nu_{e}}\left(E_{c}\right)-F_{\nu_{x}}\left(E_{c}\right)=0, \\
& F_{\bar{\nu}_{e}}\left(E_{c}\right)-F_{\bar{\nu}_{x}}\left(E_{c}\right)=0,
\end{aligned}
$$

for neutrinos and antineutrinos, respectively. A given crossing point is unstable if

$$
\begin{array}{cc}
d\left(F_{\nu_{e}}-F_{\nu_{x}}\right) / d E<0 & \text { for } \mathrm{IH} \\
d\left(F_{\nu_{e}}-F_{\nu_{x}}\right) / d E>0 & \text { for } \mathrm{NH}
\end{array}
$$

and analogously for antineutrinos.

In Fig. 1, we show the results of the flavor evolution for neutrinos (left panel) and antineutrinos (right panel) in inverted mass hierarchy. In the upper panels we show the initial and final $\nu_{e}$ and $\nu_{x}$ energy spectra, while in the lower panels we show the electron neutrino survival probability $P_{e e}$. Following the instability conditions stated above, one finds that in IH the spectral swap would develop for neutrinos around $E_{c} \simeq 13 \mathrm{MeV}$ and for antineutrinos around $E_{c} \simeq 9 \mathrm{MeV}$. The development of a spectral swap in the middle of the energy spectra produces two splits in the final $\nu$ spectra. In particular, in the $\nu_{e}$ final spectrum the swapped region is between $5 \mathrm{MeV}$ and $23 \mathrm{MeV}$, while for $\bar{\nu}_{e}$ is between $3 \mathrm{MeV}$ and $17 \mathrm{MeV}$.

In Fig. 2, the corresponding results for normal hierarchy are shown. In this case the only unstable crossing point is at $E_{c} \rightarrow \infty$ in the tail of the energy spectra, therefore the resulting $\nu$ and $\bar{\nu}$ spectra exhibit only a high-energy swap and a single split each. In this case, the split is at $E \simeq 23 \mathrm{MeV}$ for $\nu_{e}$, and at $E \simeq 17 \mathrm{MeV}$ for $\bar{\nu}_{e}$.

From this example, we realize that in the two mass hierarchies the instabilities occur around different and well-separated crossing points. This leads to spectral swaps that occur in different energy ranges for the two mass hierarchies. In fact, these ranges are nonoverlapping and almost complementary, i.e. the highenergy ends of the swaps in $\mathrm{IH}$ are the low-energy ends 


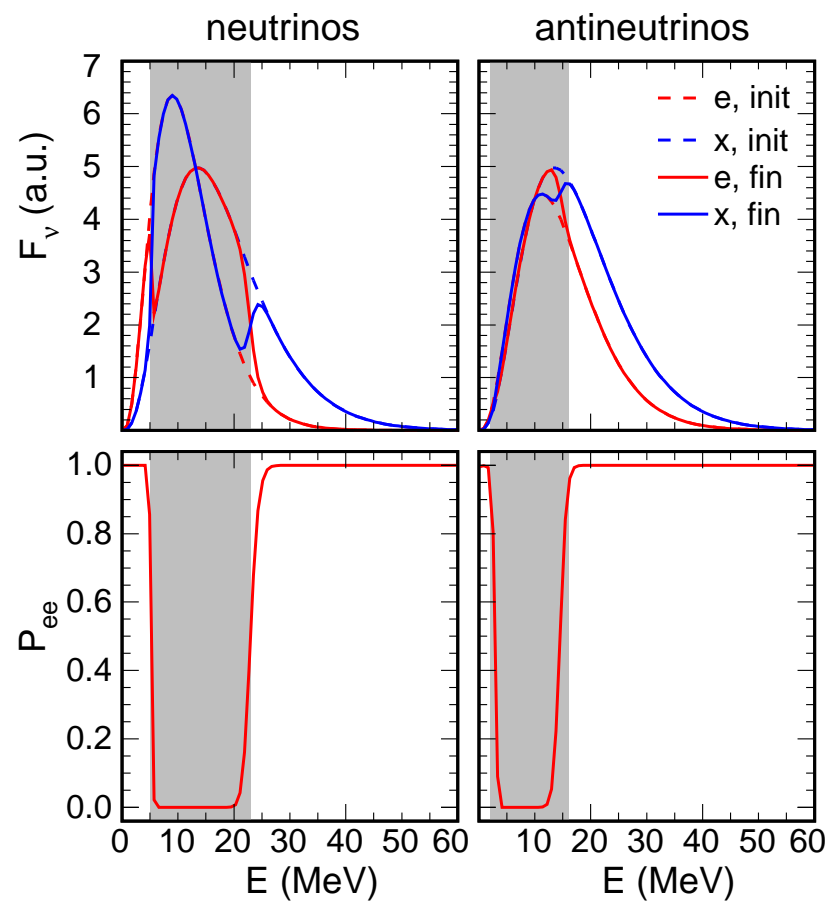

FIG. 1: Flavor evolution in $2 \nu H$-sector for neutrinos (left panels) and antineutrinos (right panels) in inverted mass hierarchy. Upper panels: the $\nu_{e}$ (red) and $\nu_{x}$ (blue) energyspectra, before (dashed curves) and after (continuous curves) the collective oscillations. Lower panels: survival probability $P_{e e}$ for electron (anti)neutrinos. The grey-regions represent the range in which the spectral swap occurs.

of the swaps in NH. This implies that if we take the $\nu$ spectra swapped by the conversions in $\mathrm{IH}$, as an input for conversions in $\mathrm{NH}$, these latter would swap also the high-energy spectrum of the electron (anti)neutrinos, giving the impression that the high-energy split has been "erased." This observation will play an important role in our understanding of the full three-flavor evolution.

\section{B. $2 \nu L$-system}

We now consider the $L$-system. Since $\Delta m_{\text {sol }}^{2}>0$, we expect a behavior similar to that of the $H$-system in normal hierarchy. However, we find that in this case no flavor conversion occurs. This has to be attributed to two reasons - insufficient growth of the instability, and lack of adiabaticity. The strength of the instability is given by the off-diagonal components in the density matrix. For a simple pendular system with energy $E$, the time-period for growth of off-diagonal components is a few times the pendular time-period $\tau_{\text {pend }}$ [8, 16]

$$
\tau_{\text {pend }} \approx \sqrt{\frac{2 E}{\Delta \tilde{m}_{\mathrm{sol}}^{2} \mu}},
$$

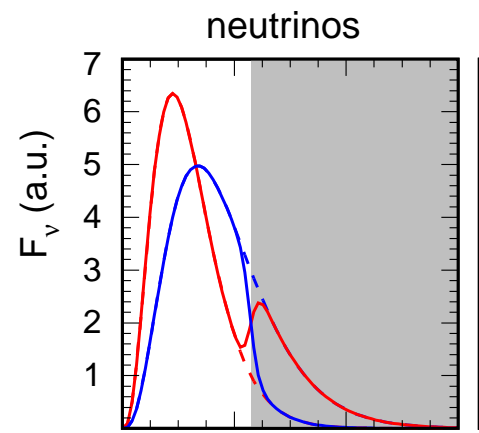

antineutrinos

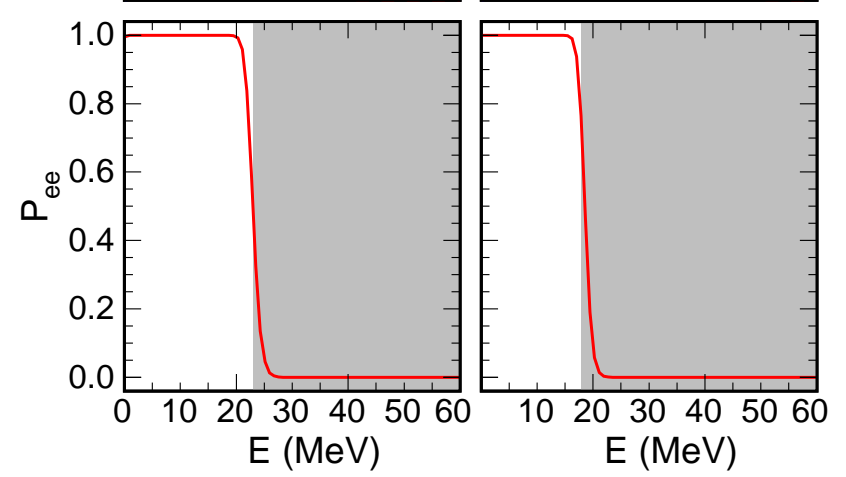

FIG. 2: Flavor evolution in the $2 \nu H$-sector for neutrinos (left panels) and antineutrinos (right panels) in normal mass hierarchy. Upper panels: the $\nu_{e}$ (red) and $\nu_{x}$ (blue) energyspectra, before (dashed curves) and after (continuous curves) the collective oscillations. Lower panels: survival probability $P_{e e}$ for electron (anti)neutrinos. The grey-regions represent the range in which the spectral swap occurs.

which scales logarithmically with the small inmedium mixing angle. This time period is roughly $\sqrt{\Delta \tilde{m}_{\mathrm{atm}}^{2} / \Delta \tilde{m}_{\mathrm{sol}}^{2}} \approx 8$ times larger for the $L$-sector, causing the instability to develop relatively slowly. The slow growth is further exacerbated by a relatively fast decrease in collective neutrino interaction strength $\mu$.

During the spectral swapping phenomenon, the spectrum near the crossing acts like an inverted pendulum [16]. The swap sweeps through the spectrum on each side of the crossing, and the modes at the edge of the swap precess at an average oscillation frequency $\kappa$ [16]. Adiabaticity requires [23]

$$
\left|\frac{d \ln \mu}{d r}\right|<\kappa .
$$

Since the collective neutrino interaction strength $\mu$ goes as $r^{-4}$, the rate at which neutrino refractive effects are decreasing is $|d \ln \mu / d r| \simeq 1 / 50 \mathrm{~km}^{-1}$ at $r \simeq 200 \mathrm{~km}$, approximately where the swapping takes place. One finds $\kappa \approx \Delta \tilde{m}_{\mathrm{sol}}^{2} / 2 E \simeq 1 / 400 \mathrm{~km}^{-1}$ for a typical energy $E \simeq$ $32 \mathrm{MeV}$ in the region of the swap, so the adiabaticity condition is not met.

Nevertheless, the $L$-system has the same instability as the $H$-system in $\mathrm{NH}$, around the crossing point $E_{c} \rightarrow$ $\infty$ of the (anti)neutrino energy spectra. Indeed, if we 

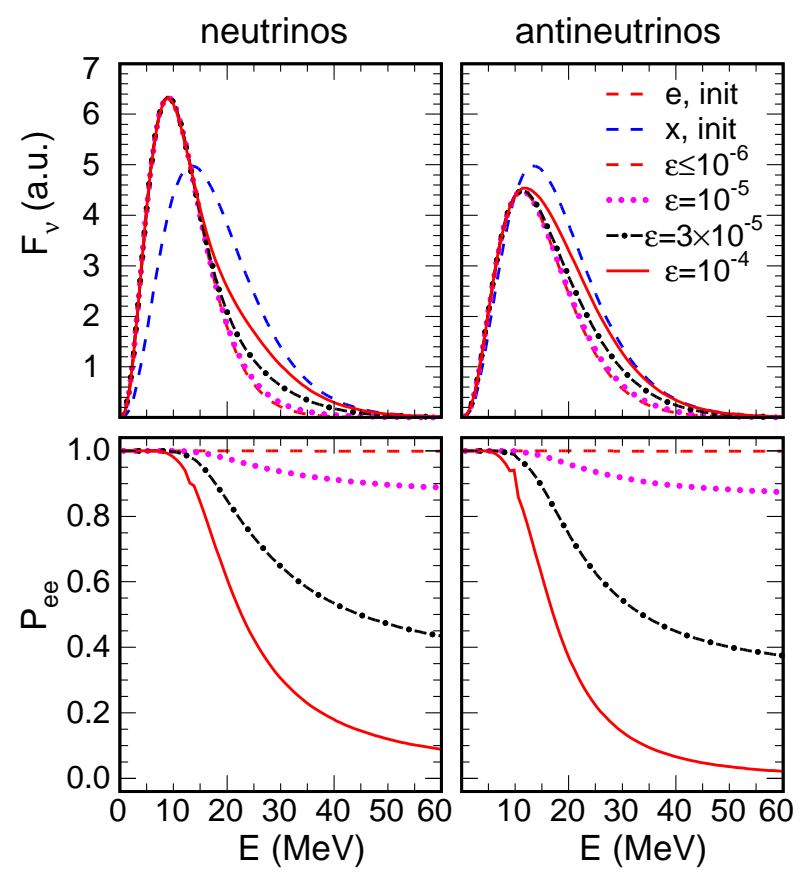

FIG. 3: Flavor evolution in the $2 \nu L$-sector for neutrinos (left panels) and antineutrinos (right panels). Upper panels: initial $\nu_{e}$ (red) and $\nu_{x}$ (blue) energy-spectra and the $\nu_{e}$ spectrum after the collective oscillations, for different values of an initial off-diagonal component of the density matrix $\left|\rho_{e x}^{0}\right|=\epsilon \times\left(\rho_{e e}^{0}+\rho_{x x}^{0}\right)$ (see the text for details). Lower panels: survival probability $P_{e e}$ for electron (anti)neutrinos.

slightly perturb the initial conditions of our $\nu$ ensemble, e.g. put explicit off-diagonal terms in the initial neutrino density matrix, the instability grows easily and leads to significant flavor conversions. ${ }^{1}$

In Fig. 3, we show the effects of these artificially triggered flavor conversions on the electron neutrinos (left panels) and antineutrinos (right panels). Upper panels show the energy spectrum, while the lower panels display the neutrino survival probability. We find that it is sufficient to take off-diagonal seeds in the density matrix

$$
\left|\rho_{e x}^{0}\right|=\epsilon \times\left(\rho_{e e}^{0}+\rho_{x x}^{0}\right),
$$

with $\epsilon \gtrsim 10^{-5}$, to produce significant flavor conversions. However, relative to the $H$-sector in the normal hierarchy, the swap is less sharp. This is naturally expected because the adiabaticity does not change significantly by taking an initial perturbation.

In Fig. 4, we show the $\left|\rho_{e x}\right|$ component of the $\nu$ density matrix for $E=32 \mathrm{MeV}$ for three cases: (i) the $H$-system with $\mathrm{NH}$, (ii) the $L$-system with $\epsilon=10^{-6}$, and (iii) the $L$ system seeded with $\epsilon=10^{-4}$. We realize that the growth

1 The speed-up of flavor instabilities under the effects of very small seeds in the initial conditions was already pointed out in 31].

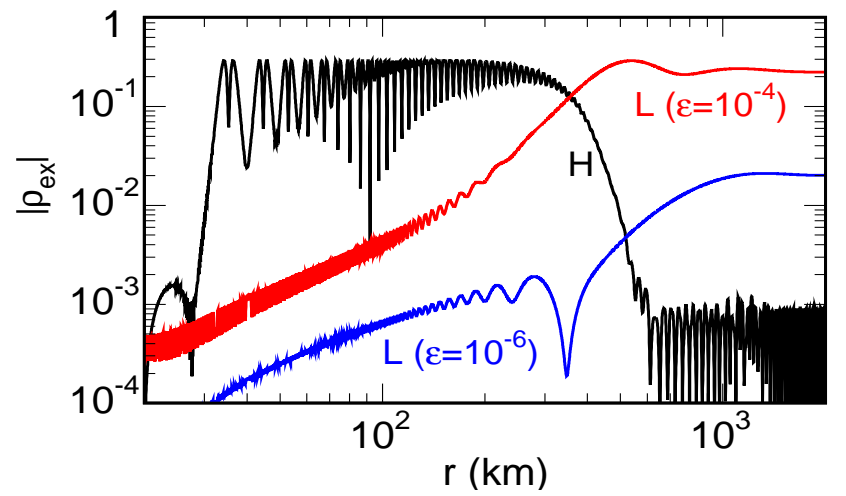

FIG. 4: Growth of the off-diagonal component $\left|\rho_{e x}\right|$ of the neutrino density matrix for the $2 \nu H$-system in normal hierarchy and for the $L$-system taking two different values of $\left|\rho_{e x}^{0}\right|=\epsilon \times\left(\rho_{e e}^{0}+\rho_{x x}^{0}\right)$. The neutrino energy is taken to be $E=32 \mathrm{MeV}$.

of the off-diagonal component is almost absent for the $L$ system with $\epsilon=10^{-6}$. Even when there are adequately large off-diagonal elements, as in the case of $\epsilon=10^{-4}$, the growth is relatively slow compared to the $H$-system, so that significant flavor changes can start only at large radius ( $r \gtrsim 300 \mathrm{~km}$ ). Consequently, due to the violation of adiabaticity, collective flavor conversions do not have enough time to develop complete splits before the effects of the neutrino-neutrino interactions become negligible.

\section{MULTIPLE SPECTRAL SPLITS IN A THREE-FLAVOR SCENARIO}

\section{A. Speed-up of the $\Delta m_{\text {sol-instability }}^{2}$}

Equipped with the insights of the previous section, we are ready to analyze the behavior of the flavor conversions in the complete three-flavor scenario. We work in the rotated basis $\left(\nu_{e}, \nu_{x}, \nu_{y}\right)=R^{T}\left(\theta_{23}\right)\left(\nu_{e}, \nu_{\mu}, \nu_{\tau}\right)$ [18]. This is equivalent to take $\theta_{23}=0$ in the neutrino mixing matrix (which makes no difference to $\nu_{e}$ and $\bar{\nu}_{e}$ evolution, if $\mu$ and $\tau$ flavors are treated identically). The vacuum Hamiltonian is written as [18]

$$
\begin{aligned}
\Omega(E) & =\frac{\Delta \tilde{m}_{\mathrm{atm}}^{2}}{2 E}\left(\begin{array}{ccc}
s_{13}^{2} & 0 & c_{13} s_{13} \\
0 & 0 & 0 \\
c_{13} s_{13} & 0 & c_{13}^{2}
\end{array}\right) \\
& +\frac{\Delta \tilde{m}_{\mathrm{sol}}^{2}}{2 E}\left(\begin{array}{ccc}
c_{13}^{2} s_{12}^{2} & c_{12} c_{13} s_{12} & -c_{13} s_{12}^{2} s_{13} \\
c_{12} c_{13} s_{12} & c_{12}^{2} & -c_{12} s_{12} s_{13} \\
-c_{13} s_{12}^{2} s_{13} & -c_{12} s_{12} s_{13} & s_{12}^{2} s_{13}^{2}
\end{array}\right),
\end{aligned}
$$

where $c_{i j}=\cos \tilde{\theta}_{i j}$ and $s_{i j}=\sin \tilde{\theta}_{i j}$. In the limit of $\theta_{13}=0$, the $\nu_{e}-\nu_{y}(H)$ and the $\nu_{e}-\nu_{x}(L)$ sectors are completely decoupled.

When the neutrino-neutrino interaction is strong, all the density matrix mode $\rho_{\mathbf{p}}$ stay pinned to each other, 
exhibiting collective flavor conversions. Now we review the factorization of the $H$ sector from other sub-leading contributions, as shown in [18]. For each energy mode, we rewrite our density matrix and Hamiltonian as

$$
\begin{aligned}
\rho & =\rho^{(0)}+\rho^{(1)}, \\
\mathbf{H} & =\mathbf{H}^{(0)}+\mathbf{H}^{(1)},
\end{aligned}
$$

where the superscript (0) refers to off-components in the $L$-sector and all diagonal components, while (1) to all others, namely

$$
\rho^{(0)}=\left(\begin{array}{ccc}
\rho_{e e} & \rho_{e x} & 0 \\
\rho_{e x}^{*} & \rho_{x x} & 0 \\
0 & 0 & \rho_{y y}
\end{array}\right),
$$

and

$$
\rho^{(1)}=\left(\begin{array}{ccc}
0 & 0 & \rho_{e y} \\
0 & 0 & \rho_{x y} \\
\rho_{e y}^{*} & \rho_{x y}^{*} & 0
\end{array}\right) .
$$

Analogous expressions hold for $\mathrm{H}^{(0)}$ and $\mathrm{H}^{(1)}$.

Note from Eq. (16) that $\mathrm{H}^{(0)}$, which contains the $e-x$ block, causes oscillations due to $\Delta m_{\text {sol }}^{2}$, while $\mathrm{H}^{(1)}$, containing the $e-y$ off-diagonal terms, gives $\Delta m_{\mathrm{atm}^{2}}^{2}$ driven oscillations. Putting this decomposition into the equations of motion, one finds [18]

$$
\begin{aligned}
& \mathrm{i} \dot{\rho}^{(0)}=\left[\mathrm{H}^{(0)}, \rho^{(0)}\right]+\left[\mathrm{H}^{(1)}, \rho^{(1)}\right], \\
& \mathrm{i} \dot{\rho}^{(1)}=\left[\mathrm{H}^{(1)}, \rho^{(0)}\right]+\left[\mathrm{H}^{(0)}, \rho^{(1)}\right] .
\end{aligned}
$$

Analogous equations hold for the antineutrinos. Note the interesting structure of the EoMs, that is an outcome of the commutation relations - $\dot{\rho}^{(0)}$ depends only on commutators $\left[\mathrm{H}^{(0)}, \rho^{(0)}\right]$ and $\left[\mathrm{H}^{(1)}, \rho^{(1)}\right]$, while $\dot{\rho}^{(1)}$ depends only on the cross-terms [18].

In a pure $2 \nu L$-system evolution, $\mathbf{H}^{(1)}$ and $\rho^{(1)}$ are zero. Thus Eq. (21) has only the first term on r.h.s., and Eq. (22) is irrelevant. Once off-diagonal components of $\rho^{(0)}$, driven by $\mathrm{H}^{(0)}$, develop asymmetrically in neutrinos and antineutrinos, the instability grows under the action of $\mathrm{H}^{(0)}$ (proportional to $\Delta m_{\mathrm{sol}}^{2}$ ), i.e. the pendular timescale is $\tau_{\text {pend }} \sim 1 / \sqrt{\omega_{\mathrm{L}} \mu}$. The addition of off-diagonal elements to $\rho^{(0)}$ kick-starts the process.

In a complete $3 \nu$ system, $\mathrm{H}^{(1)}$ is non-zero and thus produces the off-diagonal components in $\rho^{(0)}$ and $\rho^{(1)}$ more quickly. Not only do initial off-diagonal terms get generated in the $L$-sector, but also those terms grow at a much faster rate. The growth is speeded up by the second term in the equation of motion Eq. (21), i.e. by $\mathrm{H}^{(1)}$ which induces oscillations $\Delta m_{\mathrm{atm}}^{2}$-dependent at the leading order. Therefore, it leads to a growth of the instability at almost the same rate as for the $H$-system, and much faster than an isolated $L$-system.

In Fig. 5, we show the diagonal components of the neutrino density matrix $\rho_{e e}, \rho_{x x}, \rho_{y y}$ (upper panel) and the off-diagonal $\rho_{e x}, \rho_{e y}$ (lower panel) for a given energy mode with $E=32 \mathrm{MeV}$ as a function of $r$ in inverted
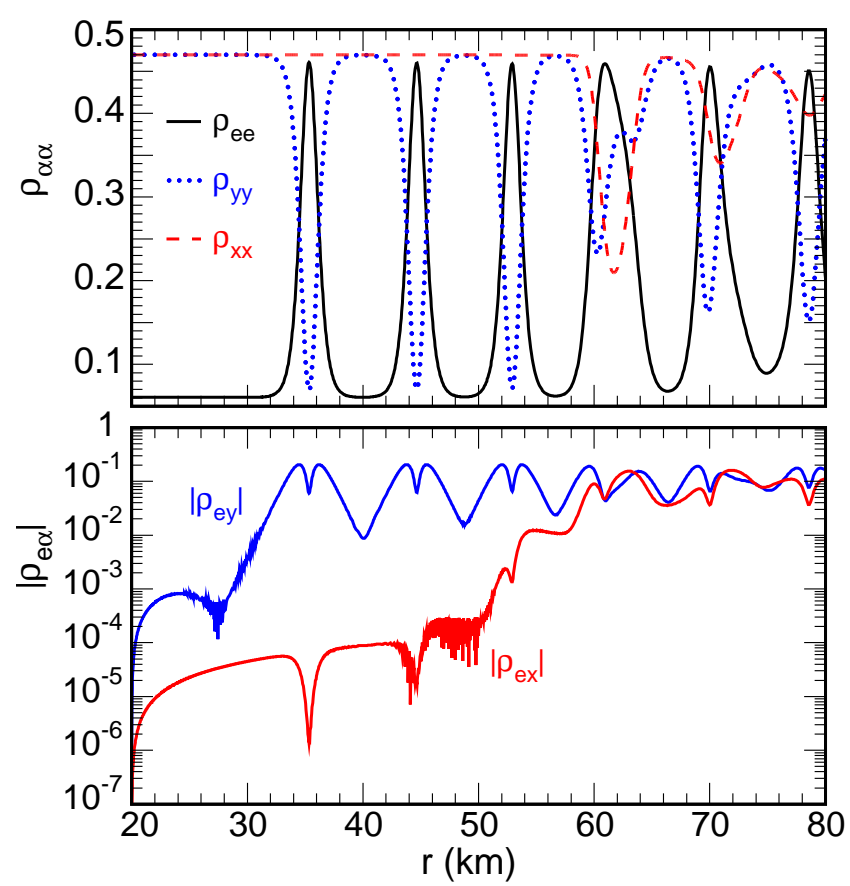

FIG. 5: Initial radial evolution of the diagonal $\rho_{e e}, \rho_{x x}$, $\rho_{y y}$ (upper panel) and off-diagonal $\rho_{e x}, \rho_{e y}$ (lower-panel) components of the neutrino density matrix for inverted mass hierarchy, shown for a neutrino energy $E=32 \mathrm{MeV}$.

mass hierarchy. The initial behavior is qualitatively similar to the one in normal hierarchy (not shown). The pendular oscillations of $\rho_{e e}$ begin to develop at $r \simeq 35 \mathrm{~km}$ for the $e-y$ sector, and proceed as pure $2 \nu$ transitions till $r \simeq 50 \mathrm{~km}$. Up to this point, $\rho_{e x}$ has not evolved significantly. All off-diagonal components increase rapidly, but the $\rho_{e x}$ starts to develop only after $\rho_{e y}$, and saturates at $r \simeq 60 \mathrm{~km}$, when $e-x$ conversions start. Note that, $\rho_{\text {ex }}$ grows faster than a $2 \nu L$-system (shown in Fig. 4), as predicted. The $\theta_{13}$-coupling between the $L$ and $H$ sectors induces three-flavor effects in the neutrino conversions: The initial kick, associated with $\Delta m_{\mathrm{atm}}^{2}$, is necessary to trigger the instability in the $L$ system.

\section{B. Inverted mass hierarchy}

In Fig. 6, we show the complete development of the $\rho_{e e}, \rho_{x x}$ and $\rho_{y y}$ components of the density matrix in inverted mass hierarchy for neutrinos (left panels) and antineutrinos (right panels) for four representative energy modes. We observe that once the conversions have been started, the different energy modes for the three neutrino species oscillate in phase, confirming the collective behavior of the flavor conversions. However, the final fate of $\rho_{e e}, \rho_{x x}$ and $\rho_{y y}$ depends on their energy. 

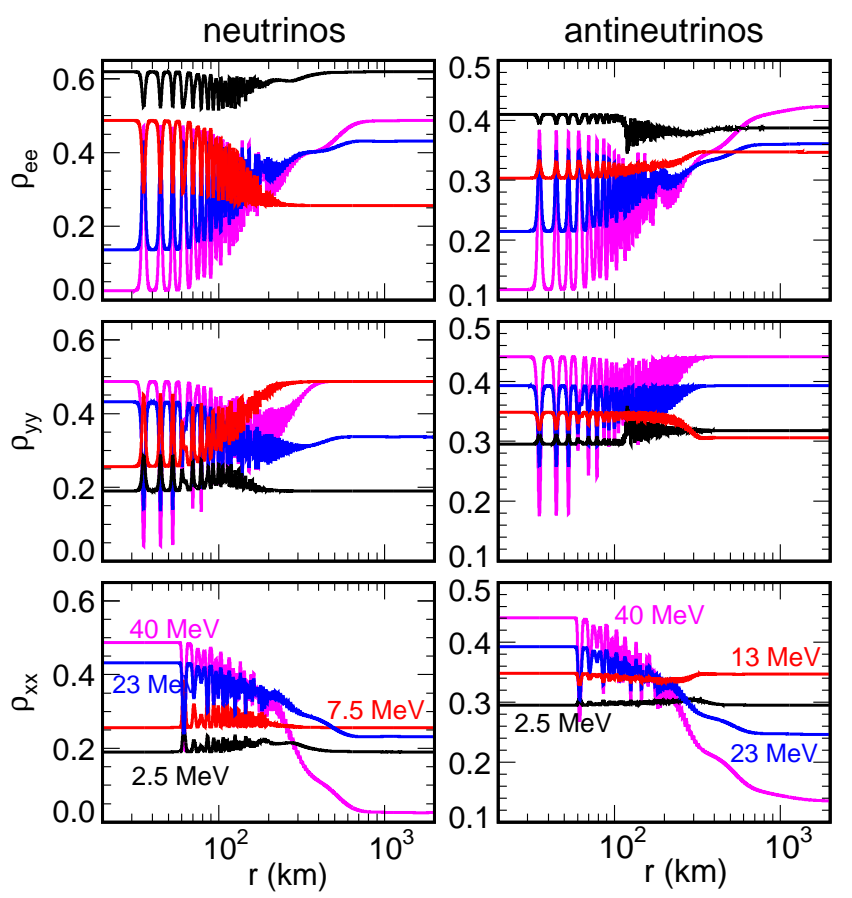

FIG. 6: Three-flavor evolution in inverted mass hierarchy. Radial evolution of the diagonal components of the density matrix $\rho$ for neutrinos (left panels) and antineutrinos (right panels) for different energy modes.

As a result of the three-flavor effects, the $\nu_{e}$ mixes with both $\nu_{x}$ and $\nu_{y}$. Therefore, the $\nu_{e}$ flavor conversions can be described in terms of the combined effects of the $L$ and $H$ two-neutrino systems. We find that the effects of the $H$-sector on the $\nu_{e}-\nu_{y}$ conversions saturate before the ones of the $L$-sector, as expected by the hierarchy between the two mass splittings. As we have discussed in Section III-A, $\Delta m_{\text {atm }}^{2}<0$ and $\Delta m_{\text {sol }}^{2}>0$ are expected to process complementary parts of the neutrino energy spectra. Therefore, their effects do not interfere in the same energy range. In particular, in the part of the neutrino energy spectra unstable under the effects of $\Delta m_{\text {atm }}^{2}$, the $\rho_{e e}$ and $\rho_{y y}$ would swap, while $\rho_{x x}$, which has been perturbed from its stable equilibrium configuration, would relax to it again. Conversely, in the part of the neutrino energy spectra unstable under the effects of $\Delta m_{\mathrm{sol}}^{2}$, the $\rho_{e e}$ and $\rho_{x x}$ swap, while $\rho_{y y}$ comes back to its initial value.

In the three-flavor space, the neutrino ensemble behaves like a pendulum. Once it is perturbed from its initial configuration, it would evolve toward its stable equilibrium position which may be different for different energy modes. In inverted mass hierarchy, the highest energy modes relax to the $x$ state, while the intermediate ones to the $y$ state, while lowest energy modes remain in the $e$ flavor.

For the modes represented in Fig. 6, we see that for

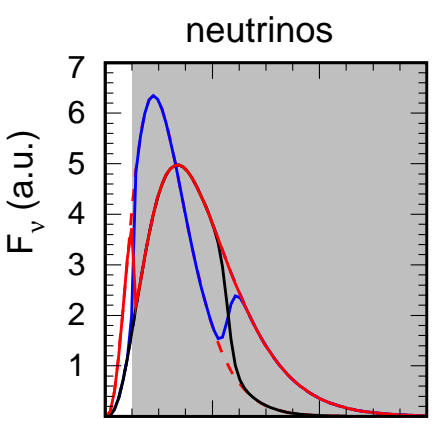

antineutrinos
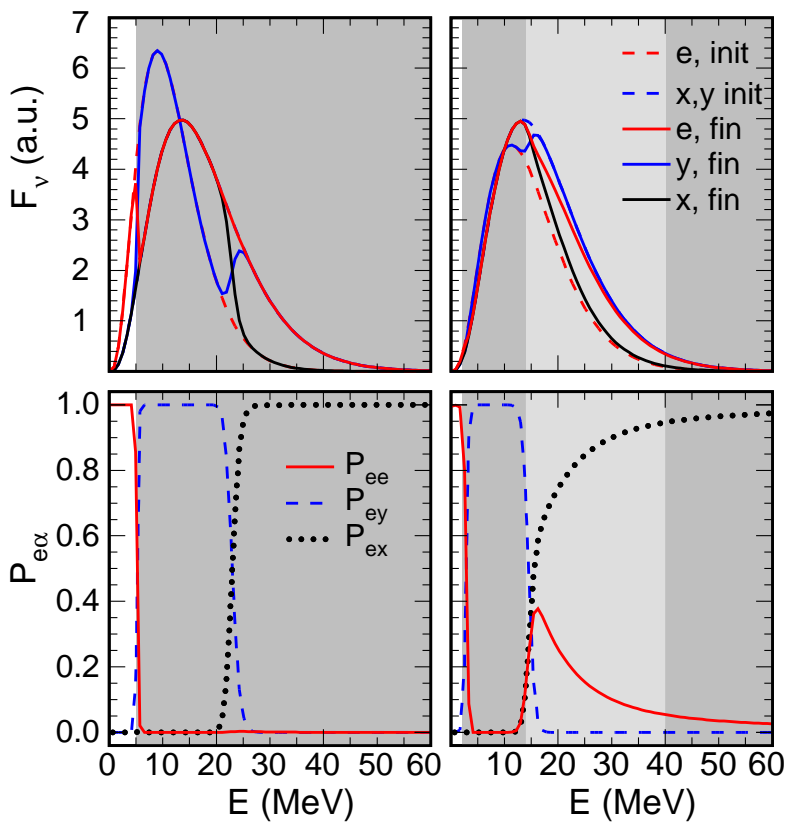

FIG. 7: Three-flavor evolution in inverted mass hierarchy for neutrinos (left panels) and antineutrinos (right panels). Upper panels: Energy spectra initially (dashed curves) and after collective oscillations (solid curves) for $\nu_{e}$ (red), $\nu_{x}$ (black) and $\nu_{y}$ (blue). Lower panels: probabilities $P_{e e}$ (solid red curve), $P_{e y}$ (dashed blue curve), $P_{e y}$ (dotted black curve). The grey bands represent the region where the spectral swaps occur. For antineutrinos the light grey band indicates the region where the spectral swap is partial (see text for details).

$E=2.5 \mathrm{MeV}$ none of the three neutrino species is affected by significant flavor changes. For $E=7.5 \mathrm{MeV}$ the flavor conversions produce a swap between $\rho_{e e}$ and $\rho_{y y}$, while $\rho_{x x}$ comes back to its original value. At $23 \mathrm{MeV}$, we are in the transition region between $e-y$ and $e-x$ conversions, therefore $\rho_{y y}$ and $\rho_{x x}$ are both partially swapped into $\rho_{e e}$. Finally, at $E=40 \mathrm{MeV}, \rho_{e e}$ and $\rho_{x x}$ exchange their initial values, while $\rho_{y y}$ returns to its original value. Therefore, the behavior of the diagonal components of the density matrix at different energies would produce a single split in the $\nu_{e}$ energy spectrum, since all the $\nu_{e}$ modes at sufficiently high energy would swap with either $\nu_{y}$ or $\nu_{x}$. Therefore, the high-energy spectral split at $E \simeq 23 \mathrm{MeV}$ observed in inverted mass hierarchy in the $2 \nu$ evolution of Sec. III-A is washed-out by the three-flavor effects. In the antineutrinos (right panels), we find an analogous behavior. At $E=2.5 \mathrm{MeV}$ $\rho_{e e}, \rho_{x x}$ and $\rho_{y y}$ are essentially unchanged. At $E=$ $13 \mathrm{MeV}$ the flavor conversions produce a swap between $\rho_{e e}$ and $\rho_{y y}$, while $\rho_{x x}$ comes back to its original value. For $E=23 \mathrm{MeV}, \rho_{y y}$ returns to its original value, while $\rho_{e e}$ and $\rho_{x x}$ tend to exchange their initial values. However, as we will discuss later, due to the the less 
adiabatic behavior around the splitting region, the $\bar{\nu}_{e}-\bar{\nu}_{x}$ swap is not complete. Finally, for $E=40 \mathrm{MeV} \rho_{e e}$ and $\rho_{x x}$ completely convert into each other and $\rho_{y y}$ is stable.

In the upper panels of Fig. 7, we show the neutrino (left panels) and antineutrino (right panels) spectra before and after the complete $3 \nu$ flavor conversions in inverted hierarchy. In the lower panel we show the corresponding $P_{e e}=P\left(\nu_{e} \rightarrow \nu_{e}\right), P_{e x}=P\left(\nu_{e} \rightarrow \nu_{x}\right)$ and $P_{e y}=P\left(\nu_{e} \rightarrow\right.$ $\left.\nu_{y}\right)$ probabilities, which can be defined approximately as the off-diagonal elements of the density matrix are small at the end of the collective evolution. Namely, these three probabilities read

$$
P\left(\nu_{e} \rightarrow \nu_{\alpha}\right)=\frac{\rho_{\alpha \alpha}^{f}-\rho_{x x}^{0}}{\rho_{e e}^{0}-\rho_{x x}^{0}},
$$

where 0 and $f$ indicate the initial and final values of $\rho$, respectively. We first consider the neutrino flavor evolution. As we have already discussed, the effects of the $L$ and $H$ systems process complementary parts of the neutrino energy spectra. Indeed, as we can see from the conversion probabilities, in the energy range where $P_{e x}=1$, we have $P_{e y}=0$ and viceversa. In particular, the solar mass splitting $\Delta m_{\mathrm{sol}}^{2}$ induces an electron survival probability $P_{e e}=0$ at high-energies, erasing the $H$-sector high-energy split at $E \simeq 23 \mathrm{MeV}$, as was pointed out in [23]. Thus, the final electron neutrino spectrum reads

$$
\begin{aligned}
F_{\nu_{e}}^{f} & =P_{e e} F_{\nu_{e}}^{0}+\left(1-P_{e e}\right) F_{\nu_{x}}^{0} \\
& \simeq \begin{cases}F_{\nu_{e}}^{0} & \text { for } E \lesssim 5 \mathrm{MeV} \\
F_{\nu_{x}}^{0} & \text { for } E \gtrsim 5 \mathrm{MeV},\end{cases}
\end{aligned}
$$

Conversely, the behavior of the $y$ and $x$ spectra is the same as the that of the non-electron species in $2 \nu$ flavor evolution in $\mathrm{IH}$ and $\mathrm{NH}$, respectively, i.e.

$$
\begin{aligned}
& F_{\nu_{y}}^{f}=P_{e y} F_{\nu_{e}}^{0}+\left(1-P_{e y}\right) F_{\nu_{x}}^{0} \\
& \simeq\left\{\begin{array}{lc}
F_{\nu_{x}}^{0} & \text { for } E \lesssim 5 \mathrm{MeV} \\
F_{\nu_{e}}^{0} & \text { for } 5 \mathrm{MeV} \lesssim E \lesssim 23 \mathrm{MeV} \\
F_{\nu_{x}}^{0} & \text { for } E \gtrsim 23 \mathrm{MeV},
\end{array}\right.
\end{aligned}
$$

and

$$
\begin{aligned}
F_{\nu_{x}}^{f} & =P_{e x} F_{\nu_{e}}^{0}+\left(1-P_{e x}\right) F_{\nu_{x}}^{0} \\
& = \begin{cases}F_{\nu_{x}}^{0} & \text { for } E \lesssim 23 \mathrm{MeV} \\
F_{\nu_{e}}^{0} & \text { for } E \gtrsim 23 \mathrm{MeV} .\end{cases}
\end{aligned}
$$

Indeed, the $\nu_{x}$ and $\nu_{y}$ spectra will be affected respectively only by the $L$ or the $H$ sector, since $x-y$ conversions are strongly suppressed as evident from the vacuum Hamiltonian in Eq. (16). One should note that while the high-energy spectral split is no longer present in the $\nu_{e}$ final energy spectrum, $\nu_{y}$ and $\nu_{x}$ final spectra still present this high-energy feature. Therefore, MSW effects in supernova, vacuum mixing and Earth effects that occur later can make the high-energy split reappear in the observable electron neutrino spectra at Earth.

Moving on to the antineutrinos, we realize that the $e-x$ swap is not sharp, due to the imperfect adiabaticity in the $L$ sector. This implies that the swap of $\bar{\nu}_{e}$ and $\bar{\nu}_{x}$ spectra at intermediate energies $(14 \mathrm{MeV} \lesssim E \lesssim 40 \mathrm{MeV})$ is not complete and this explains the "mixed" $\bar{\nu}_{e}$ spectrum, observed also in the numerical simulations in 23]. This effect has to be attributed to the low adiabaticity of the $\Delta m_{\mathrm{sol}}^{2}$-induced conversions, which is particularly visible for antineutrinos. The adiabaticity of a spectral split depends on the condition in Eq. (14). The $\bar{\nu}_{e}$ and $\bar{\nu}_{x}$ spectra are closer to each other than the corresponding neutrino spectra. This implies that in the high-energy region, where the swap takes place,

$$
\left|F_{\bar{\nu}_{e}}(E)-F_{\bar{\nu}_{x}}(E)\right| \ll\left|F_{\nu_{e}}(E)-F_{\nu_{x}}(E)\right| .
$$

In the final phases of the swapping dynamics, the neutrino and antineutrino spectra evolve quite independently, and the precession frequencies of the two blocks are not governed by a common $\mu$, but by individual $\mu$ 's proportional to the flux differences in Eq. (27). They behave essentially as two uncoupled oscillators because the neutrino-neutrino interaction $\mu$ is now smaller than the frequency difference of the two blocks. One can see this clearly in the numerical simulations of Ref. [16], shown at 32]. The frequency $\tau_{\text {pend }}^{-1} \approx \sqrt{\omega \mu}$ is lower for antineutrinos [16], and thus adiabaticity tends to be broken more severely for antineutrinos.

\section{Normal mass hierarchy}

In Fig. 8, we show the complete development of the $\rho_{e e}$, $\rho_{x x}$ and $\rho_{y y}$ components of the density matrix in normal hierarchy for neutrinos (left panels) and for antineutrinos (right panels) for different energy modes. For both neutrinos and antineutrinos, the swapping dynamics occurs mainly between the $\rho_{e e}$ and the $\rho_{y y}$, while $\rho_{x x}$ experiences only nutations around its initial equilibrium value, before relaxing completely to it when the flavor conversions are saturated. Therefore, the flavor evolution is close to the $2 \nu H$-case in normal hierarchy discussed in Sec. III-A. Indeed, both $\Delta m_{\text {atm }}^{2}$ and $\Delta m_{\text {sol }}^{2}$ are positive. Therefore, the $L$-sector would behave as a replica of the $H$-sector, but with a smaller mass splitting. In this condition, both $H$ and $L$ sectors process the same regions of the electron neutrino energy spectra. However, the hierarchy between the two mass splittings produces the dominance of the $\nu_{e}-\nu_{y}$ swaps, while conversion effects in the $\nu_{e}-\nu_{x}$ sector remain inhibited by adiabaticity violation. The only region where the $L$-instability can compete with $H$ is very close to the split. In particular, for low-energy neutrino modes $(E=2.5,18 \mathrm{MeV}$ in Fig. 8) the $\rho_{e e}$ comes back to its initial value, while at higher energies $(E=23,40 \mathrm{MeV})$ exchanges its initial value with $\rho_{y y}$. For the antineutrinos, $\rho_{e e}$ remains at its initial value for the modes at $E=2.5 \mathrm{MeV}$. 

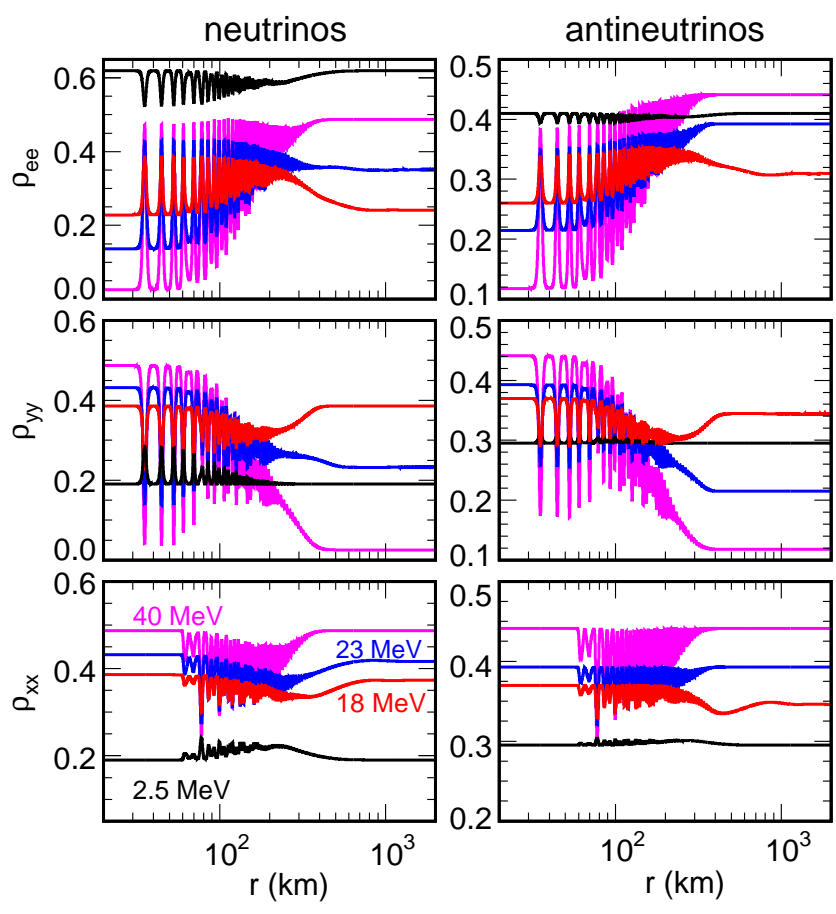

FIG. 8: Three-flavor evolution in normal mass hierarchy. Radial evolution of the diagonal components of the density matrix $\rho$ for neutrinos (left panels) and antineutrinos (right panels) for different energy modes.

At $E=18 \mathrm{MeV}$, we are around the splitting region where the adiabaticity in the $H$-system is more severely violated, and the $e-y$ conversions are not complete. Under these conditions, the $L$ instability can also play a role, producing a weak swap in $\rho_{x x}$. Finally, at $E=40 \mathrm{MeV}$, conversions again occur only between $e$ and $y$ states, producing a complete swap of the initial $\rho_{e e}$ and $\rho_{y y}$ values.

In Fig. 9, we show the neutrinos (left panels) and antineutrino (right panels) spectra before and after the complete $3 \nu$ flavor conversions in NH. In the lower panels we represent the corresponding $P_{e e}, P_{e x}$ and $P_{e y}$ probabilities. Once more, we realize that the flavor evolution can be mostly described in terms of twoflavor $\nu_{e}-\nu_{y}$ transitions, while the role of $\nu_{e}-\nu_{x}$ conversions is subleading. The spectral splitting features are remarkably similar to the ones found for the $2 \nu \mathrm{H}$ system in normal hierarchy (Sec. III-A). In particular, the $\nu_{e}$ spectrum presents a single split at $E \simeq 23 \mathrm{MeV}$ and $\bar{\nu}_{e}$ spectrum around $E \simeq 17 \mathrm{MeV}$. Only close to the splitting regions, we find subleading effects associated with $\nu_{e}-\nu_{x}$ conversions.

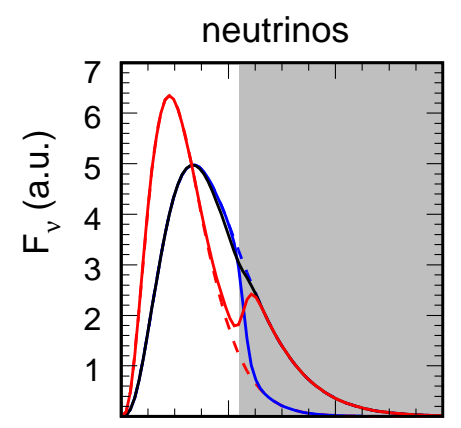

antineutrinos
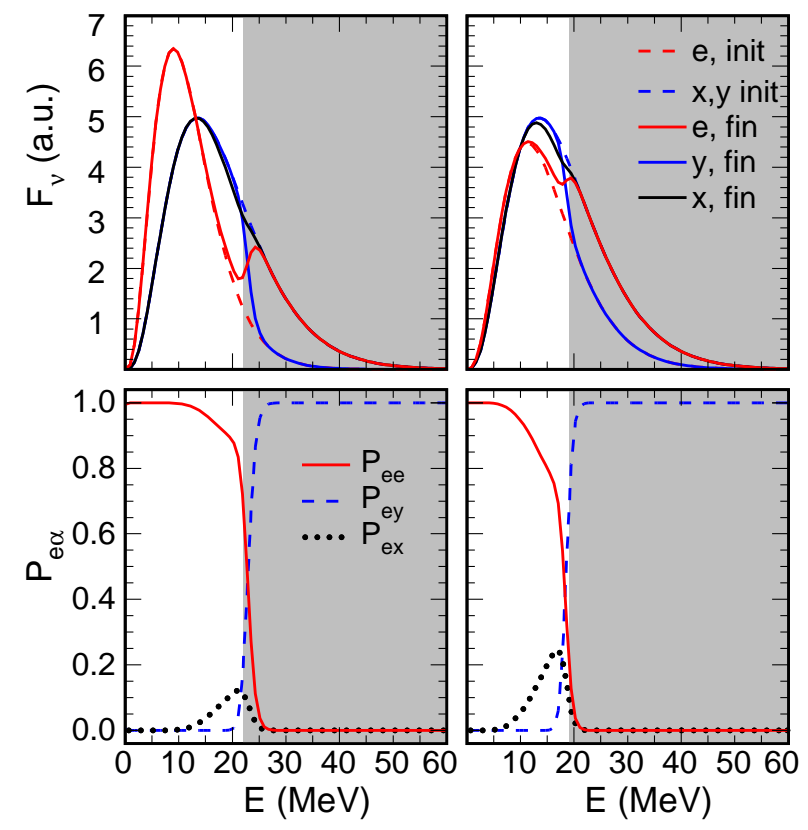

FIG. 9: Three-flavor evolution in normal mass hierarchy for neutrinos (left panels) and antineutrinos (right panels). Upper panels: Energy spectra initially (dashed curves) and after collective oscillations (solid) for $\nu_{e}$ (red), $\nu_{x}$ (black) and $\nu_{y}$ (blue). Lower panels: corresponding probabilities $P_{e e}$ (solid red curve), $P_{e y}$ (dashed blue curve), $P_{e y}$ (dotted black curve). The grey bands represent the region where the spectral swaps occur (see text for details).

\section{CONCLUSIONS}

Collective neutrino flavor conversions in supernovae, associated with neutrino-neutrino interactions, have been recognized to induce peculiar spectral swaps among the different neutrino species. The development of these features is associated with instabilities in the flavor space. In particular, these instabilities would develop around the crossing points of the original SN neutrino spectra. Then, the neutrino mass hierarchy determines if a crossing point is unstable under the effects of the collective oscillations. A particularly intriguing case is the one in which the original SN neutrino fluxes exhibit an ordering with $\Phi_{\nu_{x}}^{0} \gtrsim \Phi_{\nu_{e}}^{0} \gtrsim \Phi_{\bar{\nu}_{e}}^{0}$, possible during the cooling phase. In this case, the two-flavor study realized by [16], found the occurrence of multiple spectral splits for both neutrinos and antineutrinos, depending on the neutrino mass hierarchy. A recent numerical exploration of this case performed in [23], has found that when threeflavor effects are taken into account in inverted mass hierarchy, the high-energy spectral swaps observed in the $2 \nu$ evolution are erased by effects related to $\Delta m_{\mathrm{sol}}^{2}$.

Motivated by this intriguing result, in our paper we have performed a detailed study of the three-flavor 
effects in the collective oscillations for supernova neutrino spectra typical of the cooling phase. We have found that the effects of $\Delta m_{\text {sol }}^{2}$ in the three-flavor evolution are important only in inverted mass hierarchy. In this case, the presence of $\Delta m_{\text {sol }}^{2}$ gives rise to instabilities in regions of the neutrino energy spectra that were stable under the two-flavor evolution governed by $\Delta m_{\text {atm }}^{2}$ and $\theta_{13}$. Therefore, the combinations of these two different instabilities would produce a wash-out of the high-energy splitting features in the $\nu_{e}$ and $\bar{\nu}_{e}$ spectra. Conversely, in normal mass hierarchy the three-flavor instabilities and the two-flavor one act in the same regions of the neutrino energy spectrum, leading only to minor departures from the two-flavor evolution. Essentially, the system behaves like a pendulum in $3 \nu$ flavor space. It can topple towards either the $\nu_{y}$ state or the $\nu_{x}$ state. In inverted hierarchy, when the $H$ and $L$ instabilities are in different regions of energy, the pendulum topples towards $\nu_{y}$ for the $H$-instability, and towards $\nu_{x}$ for the $L$-instability. In normal hierarchy, when the instabilities are in the same region of energy, the pendulum topples towards $\nu_{y}$, as the $L$-instability is relatively non-adiabatic. As a consequence, in inverted mass hierarchy the electron (anti)neutrino spectrum at the end of the collective oscillations would present only a very low-energy $(E \lesssim$ $5 \mathrm{MeV}$ ) splitting feature, being completely swapped to the original non-electron spectra at higher energies.

We wish to emphasize that the high-energy splitting features may survive in the observable electron (anti)neutrino spectrum at Earth, even in inverted hierarchy. Indeed, MSW matter effects in SN, vacuum mixing and Earth effects would further mix the $\nu_{e}$ and $\nu_{x}$ spectra. The non-electron spectrum at the end of the collective oscillations, still contains high-energy splitting features, since for the non-electron species the collective flavor conversions have occurred essentially as in the twoflavor case. Therefore, especially for neutrinos, which have sharper spectral swaps, the electron neutrino signal at Earth could still present observable splitting features at high energy. Also, due to the lower neutrino luminosity at sufficiently late times, the initial collective interaction strength $\mu_{0}$ can be somewhat lower than is assumed in Ref. 23] and this work. We found that in certain regions of the spectral parameter space, reducing $\mu_{0}$ by a factor of 10 makes the three-flavor effect disappear due to a stronger adiabaticity violation in the $L$ sector. In principle, this effect could produce interesting signatures in the time evolution of the SN neutrino signal.

In conclusion, the non-linear equations that govern the flavor evolution of neutrinos emitted during a stellar collapse are a continuous source of surprises and new effects. During this last year, dramatic changes have occurred in the picture consolidated after the initial exploration of collective supernova neutrino oscillations. The discovery of this new three-flavor effect is the most recent of these changes. After our study, it appears that its impact on the collective neutrino flavor conversions is conceptually and quantitatively well under control.

\section{Acknowledgments}

We thank Alois Kabelschacht for fruitful discussions during the development of our work and for a careful reading our manuscript. R.T. thanks Andreu EstebanPretel for useful discussions. B.D. thanks Alexander Friedland for useful correspondence. B.D. and I.T. were partly supported by the Deutsche Forschungsgemeinschaft under grant TR-27 "Neutrinos and Beyond" and the Cluster of Excellence "Origin and Structure of the Universe" (Munich and Garching). The work of I.T. has been partly supported by the Italian MIUR and INFN through the "Astroparticle Physics" research project. Her stay in Munich has been partly supported by a junior fellowship awarded by the Italian Society of Physics (Borsa SIF "Antonio Stanghellini").
[1] G. G. Raffelt, "Supernova neutrino observations: What can we learn?," astro-ph/0701677.

[2] A. Dighe, "Physics potential of future supernova neutrino observations," J. Phys. Conf. Ser. 136, 022041 (2008) arXiv:0809.2977 [hep-ph]].

[3] A. S. Dighe and A. Yu. Smirnov, "Identifying the neutrino mass spectrum from the neutrino burst from a supernova," Phys. Rev. D 62, 033007 (2000) hep-ph/9907423.

[4] L. Wolfenstein, "Neutrino Oscillations In Matter," Phys. Rev. D 17, 2369 (1978); S. P. Mikheev and A. Yu. Smirnov, "Resonance Enhancement Of Oscillations In Matter And Solar Neutrino Spectroscopy," Yad. Fiz. 42, 1441 (1985) [Sov. J. Nucl. Phys. 42, 913 (1985)].

[5] A. Dighe, "Supernova neutrino oscillations: what do we understand?," J. Phys. Conf. Ser. 203, 012015 (2010) arXiv:0912.4167 [hep-ph]].

[6] H. Duan, G. M. Fuller and Y. Z. Qian, "Collective
Neutrino Oscillations," arXiv:1001.2799 [hep-ph]].

[7] H. Duan, G.M. Fuller, J. Carlson and Y.Z. Qian, "Simulation of coherent non-linear neutrino flavor transformation in the supernova environment. I: Correlated neutrino trajectories," Phys. Rev. D 74, 105014 (2006) astro-ph/0606616.

[8] S. Hannestad, G. G. Raffelt, G. Sigl and Y. Y. Y. Wong, "Self-induced conversion in dense neutrino gases: Pendulum in flavor space," Phys. Rev. D 74, 105010 (2006); Erratum ibid. 76, 029901 (2007) astro-ph/0608695.

[9] T. Totani, K. Sato, H. E. Dalhed and J. R. Wilson, "Future detection of supernova neutrino burst and explosion mechanism," Astrophys. J. 496, 216 (1998) astro-ph/9710203.

[10] R. Buras, H. T. Janka, M. T. Keil, G. G. Raffelt and M. Rampp, "Electron-neutrino pair annihilation: A new source for muon and tau neutrinos in supernovae," Astrophys. J. 587, 320 (2003) astro-ph/0205006. 
[11] M. T. Keil, G. G. Raffelt and H. T. Janka, "Monte Carlo study of supernova neutrino spectra formation," Astrophys. J. 590, 971 (2003) astro-ph/0208035.

[12] G. G. Raffelt and A. Yu. Smirnov, "Self-induced spectral splits in supernova neutrino fluxes," Phys. Rev. D 76, 081301 (2007) arXiv:0705.1830 [hep-ph]].

[13] H. Duan, G. M. Fuller and Y. Z. Qian, "A simple picture for neutrino flavor transformation in supernovae," Phys. Rev. D 76, 085013 (2007) arXiv:0706.4293 [astro-ph]].

[14] G. L. Fogli, E. Lisi, A. Marrone and A. Mirizzi, "Collective neutrino flavor transitions in supernovae and the role of trajectory averaging," JCAP 0712, 010 (2007) arXiv:0707.1998 [hep-ph]].

[15] G. L. Fogli, E. Lisi, A. Marrone, A. Mirizzi and I. Tamborra, "Low-energy spectral features of supernova (anti)neutrinos in inverted hierarchy," Phys. Rev. D 78, 097301 (2008) arXiv:0808.0807 [hep-ph]].

[16] B. Dasgupta, A. Dighe, G. G. Raffelt and A. Y. Smirnov, "Multiple Spectral Splits of Supernova Neutrinos," Phys. Rev. Lett. 103, 051105 (2009) arXiv:0904.3542 [hep-ph].

[17] G. Fogli, E. Lisi, A. Marrone and I. Tamborra, "Supernova neutrinos and antineutrinos: ternary luminosity diagram and spectral split patterns," JCAP 0910, 002 (2009) arXiv:0907.5115 [hep-ph]].

[18] B. Dasgupta and A. Dighe, "Collective three-flavor oscillations of supernova neutrinos," Phys. Rev. D 77, 113002 (2008) arXiv:0712.3798 [hep-ph]].

[19] A. Esteban-Pretel, S. Pastor, R. Tomas, G. G. Raffelt and G. Sigl, "Mu-tau neutrino refraction and collective three-flavor transformations in supernovae," Phys. Rev. D 77, 065024 (2008) arXiv:0712.1137 [astro-ph]].

[20] G. Fogli, E. Lisi, A. Marrone and I. Tamborra, "Supernova neutrino three-flavor evolution with dominant collective effects," JCAP 0904, 030 (2009) arXiv:0812.3031 [hep-ph]].

[21] J. Gava and C. Volpe, "Collective neutrinos oscillation in matter and CP-violation," Phys. Rev. D 78, 083007 (2008) arXiv:0807.3418 [astro-ph]].

[22] B. Dasgupta, G. G. Raffelt and I. Tamborra, Phys. Rev.
D 81, 073004 (2010) arXiv:1001.5396 [hep-ph]].

[23] A. Friedland, "Self-refraction of supernova neutrinos: mixed spectra and three-flavor instabilities," Phys. Rev. Lett. 104, 191102 (2010) arXiv:1001.0996 [hep-ph]].

[24] G. Sigl and G. Raffelt, "General kinetic description of relativistic mixed neutrinos," Nucl. Phys. B 406, 423 (1993).

[25] A. Esteban-Pretel, S. Pastor, R. Tomàs, G. G. Raffelt and G. Sigl, "Decoherence in supernova neutrino transformations suppressed by deleptonization," Phys. Rev. D 76, 125018 (2007) arXiv:0706.2498 [astro-ph]].

[26] B. Dasgupta, A. Dighe, A. Mirizzi and G. G. Raffelt, "Collective neutrino oscillations in non-spherical geometry," Phys. Rev. D 78, 033014 (2008) arXiv:0805.3300 [hep-ph]].

[27] M. C. Gonzalez-Garcia, M. Maltoni and J. Salvado, "Updated global fit to three neutrino mixing: status of the hints of theta13 i 0," JHEP 1004, 056 (2010) arXiv:1001.4524 [hep-ph]].

[28] A. Esteban-Pretel, A. Mirizzi, S. Pastor, R. Tomas, G. G. Raffelt, P. D. Serpico and G. Sigl, "Role of dense matter in collective supernova neutrino transformations," Phys. Rev. D 78, 085012 (2008) arXiv:0807.0659 [astro$\mathrm{ph}]$.

[29] H. Duan, G. M. Fuller and Y. Z. Qian, "Stepwise Spectral Swapping with Three Neutrino Flavors," Phys. Rev. D 77, 085016 (2008) arXiv:0801.1363 [hep-ph]].

[30] J. Gava, J. Kneller, C. Volpe and G. C. McLaughlin, "A dynamical collective calculation of supernova neutrino signals," Phys. Rev. Lett. 103, 071101 (2009) arXiv:0902.0317 [hep-ph]].

[31] R. F. Sawyer, "'Classical' instabilities and 'quantum' speed-up in the evolution of neutrino clouds," hep-ph/0408265.

[32] B. Dasgupta, A. Dighe, G. G. Raffelt and A. Y. Smirnov, Animated figures available at http://www.mppmu.mpg.de/supernova/multisplits 